\section{RECURRENT HEMATOSPERMIA DUE TO ASPIRIN}

Sir,

The presence of blood in the semen is called hematospermia and represents $1 \%$ of all andrologic and urologic symptoms. ${ }^{[1]}$ Hematospermia is an uncommon clinical condition that usually follows a painless, benign and self-limited course; but it can be a source of considerable fear and anxiety that is uncommonly associated with significant underlying pathology and is mostly considered to be idiopathic in nature. ${ }^{[2]}$ Hematospermia is classified by its cause, which may be an inflammation or infection, an obstruction or cyst, a vascular abnormality, a systemic disease, a tumor or an idiopathic condition..$^{[3]}$ Management with appropriate clinical evaluation, watchful waiting, and reassurance often suffices without further diagnostic workup or treatment. [2]

A 32-year-old man referred to our hospital in Gorgan, north of Iran; he presented with recurrent painless hematospermia of 18 months' duration. He did not have any history of urinary tract infections; sexually transmitted diseases; prolonged and intense masturbation or sexual intercourse; hypertension; renal, liver or bleeding disorders; abdominal trauma or surgery. He did not report erectile dysfunction (International Index of Erectile Function score, 25). The findings of general physical examination of the patient were normal. No evidence of trauma or self-instrumentation in the urogenital region was found. The prostate was normal in size and consistency, and no abnormal masses were palpable on digital examination. External genitalia, testes, epididymis, cord and penile urethra were normal. Seminal vesicles were not palpable. The urethral meatus was evaluated, and no evidence of trauma, condylomata and phimosis was found. Hematologic studies (PT, PTT, CBC and ESR), urinalysis, urine culture, semen analysis and semen culture were done, and none of these revealed any abnormality. Results of PSA test, mycobacterial cultures, urethral cultures for gonorrhea and chlamydia, transrectal ultrasound of the prostate and abdominopelvic sonography were normal. Empirical treatment with an antibiotic (quinolone) and an anti-inflammatory medication (selective COX-2 inhibitor) was administered, to which there was no response. The patient was evaluated for epididymitis, prostitis, urethritis, HIV, condylomata, urinary stones, liver cirrhosis, arterial hypertension, genitourinary TB and hematologic disease, and he was normal in all these assessments; so the specialists reassured him that this condition was benign and self limited. Finally, he reported ASA consumption due to hemoconcentration 2 years ago. ASA effect as a causation of hematospermia was suspected. We carried out coagulation tests (PT, PTT, BT, and CT), which, again, were normal except for BT, which was slightly prolonged. So ASA was discontinued and hematospermia stopped and was not found to appear during the follow-ups in the following 6 months. For confirmation of the mentioned diagnosis, ASA was restarted and hematospermia reappeared. We can affirm this cause-and-effect relationship to some extent because of the reappearance of 
hematospermia when ASA was restarted. The etiology in relation to this finding may be that hematospermia could have been caused by micro-trauma after ejaculatory contractions, and aspirin may have been a contributing factor by damaging the integrity of a congested mucosa or interfering with platelet function, which could have produced hematospermia. The importance of this case presentation is that no similar reports about the side effects of aspirin are found in the literature according to our search.

LAILY NAJAFI, AMIR HUSSEIN NOOHI ${ }^{1}$

Departments of Clinical Research and 'Pediatrics, Golestan University of Medical Sciences, Gorgan, Iran

Correspondence:

Dr. Amir hussein Noohi, Iran-Gorgan-Taleghany hospital Gorgan Iran Email: a_noohi2000@yahoo.com

\section{REFERENCES}

1. Polito M, Giannubilo W, d'Anzeo G, Muzzonigro G. Hematospermia: Diagnosis and treatment. Arch Ital Urol Androl 2006;78:82-5.

2. Torigian DA, Ramchandani P. Hematospermia: imaging findings. Abdom Imaging 2007;32:29-49.

3. Labadie P, Hellstrom WJ. Hematospermia diagnosis, evaluation, and clinical relevance. Contem Uro Arch 1999;11:125-9.

DOI: $10.4103 / 0019-5359.53397$ 